\title{
Work In Progress: Incorporating Global Context into a Biomechanics Course through Service-learning Collaboration
}

\section{Dr. Sarah Ilkhanipour Rooney, University of Delaware}

Sarah I. Rooney is an Assistant Professor and Director of the Undergraduate Program in the Biomedical Engineering department at the University of Delaware, where she seeks to bring evidence-based teaching practices to the undergraduate curriculum. She received her B.S.E. (2009) and M.S.E. (2010) in Biomedical Engineering from the University of Michigan (Ann Arbor) and her Ph.D. (2015) in Bioengineering from the University of Pennsylvania.

\section{Ms. Krystina Callahan \\ Dr. Kimberly L Bothi, University of Delaware}

Dr. Kim Bothi has a multidisciplinary background in engineering and social sciences, with research and consulting experience across a range of developing country contexts. She earned a Ph.D. in global community-based resource management from Cornell University (2012), and holds earlier degrees in environmental engineering from Cornell (MS, 2007) and McGill University (BSc.Eng., 2000). As Director of Global Engineering in the University of Delaware's College of Engineering, Kim is responsible for expanding opportunities for students, staff and faculty to engage in cross-disciplinary, globally-minded research and academic programming. In 2016 and 2017, Kim co-led UD's Mandela Washington Fellowship Civic Leadership Institute as part of President Obama's Young African Leaders Initiative. She advises the UD student chapter of Engineers Without Borders, which links student teams with community partners abroad to implement sustainable engineering projects. 


\section{Work In Progress: Incorporating Global Context to a Biomechanics Course through Service-Learning Collaboration}

\section{Introduction}

ABET, the accrediting body for engineering programs, expects undergraduate engineering programs to prepare students with "an ability to apply engineering design to produce solutions that meet specified needs with consideration of public health, safety, and welfare, as well as global, cultural, social, environmental, and economic factors" (outcome 2) as well as "an ability to recognize ethical and professional responsibilities in engineering situations and make informed judgments, which must consider the impact of engineering solutions in global, economic, environmental, and societal contexts" (outcome 4) [1]. Both of these outcomes require that students consider the global impact of engineering. Global engineering skills are critical to prepare students for the realities of American competitiveness in global markets [2], and to build students' interdisciplinary, cross-cultural capacities to tackle grand challenges, a sentiment enthusiastically supported by over 120 engineering deans in a letter to President Obama in 2015 [3]. Biomedical engineering undergraduate curricula often have little room to add stand-alone courses in global engineering or provide equitable opportunities for learning abroad, and instead, these topics may be addressed through core courses. Here we present a unique framework to develop and implement globally-minded curricular tools in a biomechanics course. Our goal was to combine the global expertise of members of the undergraduate service learning organization, Engineers without Borders (EWB), with traditional statics and mechanics content to develop problems that would 1) help students identify the relationship between engineering concepts and global challenges, 2) help students understand how engineers can contribute to solving complex, global challenges, and 3) increase student interest in global engineering applications.

\section{Methods}

Bioengineering Mechanics I is a fall semester, junior-level, core course in our biomedical engineering undergraduate curriculum. In this course, students learn how to apply a systematic problem-solving approach in statics (forces, moments, equilibrium) and mechanics of materials (internal forces/moments, stress, strain, axial loading, torsion, bending) with applications to the human body, biologic systems, and biomedical devices. "Honors" students are expected to complete a project in addition to the typical coursework.

In fall 2016, one honors student enrolled in Bioengineering Mechanics I collaborated with members of EWB to develop two statics/mechanics homework problems inspired by current EWB projects in partner communities across the developing world: 1) "Meet Aminah"- static equilibrium of supporting a bucket of water on one's head and 2) "Shoulder Pole"- beam bending of carrying supplies with a shoulder yoke. In "Meet Aminah," students were introduced to the scarcity of safe drinking water and how the challenge of water collection disproportionately impacts women and young girls on a daily basis. The students learned how far someone may walk to fetch safe water and how this responsibility can interfere with school attendance and educational achievement. In "Shoulder Pole," students were introduced to how the most efficient form of travel is often by foot in locations where roads are regularly impassable or it is difficult to arrange transport for smaller loads. Students learned how many 
Eastern Asian cultures use bamboo (a sustainable resource) shoulder poles to transport basic goods and supplies. These two homework problems were implemented in fall 2017 in place of other homework problems, requiring no additional work for the fall 2017 cohort. The honors student spent an estimated 20 hours total in developing the 2 problems.

This study was classified as exempt from IRB review, per University and federal guidelines. Students enrolled in Bioengineering Mechanics I in fall 2017 completed an anonymous pre(beginning of semester) and post- (end of semester) survey to indirectly assess the aforementioned goals. Students were asked 3 Likert scale questions (1- strongly agree, 2somewhat agree, 3- neither agree nor disagree, 4- somewhat disagree, 5- strongly disagree):

- Q1: The relationship between engineering statics/mechanics concepts and global challenges is clear to me.

- Q2: I understand how biomedical engineers can contribute to solving complex, global challenges.

- Q3: I am interested in global engineering applications.

Comparisons between pre- and post-survey responses for the Likert scale questions were made with a Mann-Whitney U test for nonparametric data in R. Significance was set at $p \leq 0.05$. Students were also asked 2 free-response questions:

- How can biomedical engineers contribute to solving complex, global challenges?

- Provide an example of engineering statics/mechanics concepts applied to a real-world scenario in a culture different from your own.

After completion of each homework problem, students completed an anonymous survey on how effective that specific problem was in addressing the goals:

- Q4: The problem helped me understand how engineering statics/mechanics concepts apply globally to real-world scenarios [Likert 1-5]. Why do you say that?

- Q5: The problem helped me understand how engineers contribute to solving global challenges [Likert 1-5]. Why do you say that?

- Q6: The problem increased my interest in global applications of engineering [Likert 1-5]. Why do you say that?

\section{Results}

In the pre-survey $(\mathrm{N}=56), 86 \%$ of students agreed (strongly or somewhat) with Q1 that the relationship between engineering statics/mechanics concepts and global challenges is clear to them. In the post-survey, $98 \%$ agreed $(\mathrm{N}=50)$ with Q1, representing a statistically significant $(p=0.04)$ shift in the distribution (Figure 1). No significant differences between preand post-survey scores were found for Q2 (pre: 98\% agreed, post: 100\% agreed) or Q3 (pre: 80\% agreed, post: 80\% agreed).
Q1: The relationship between engineering statics/mechanics concepts and global challenges is clear to me.

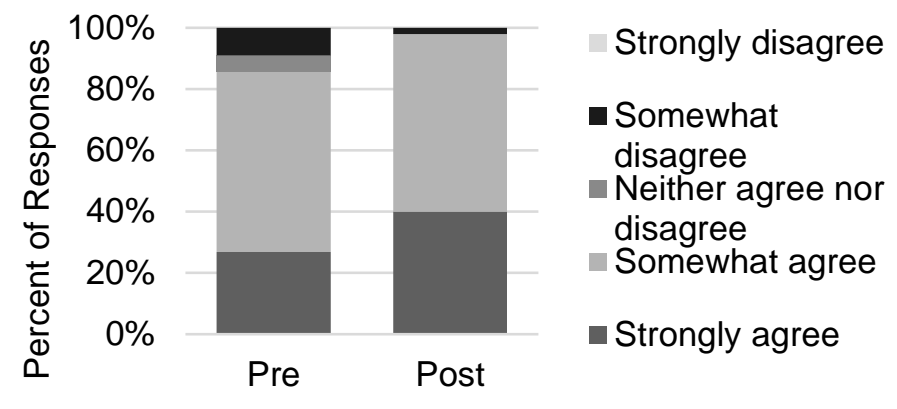

Figure 1. Post-survey responses indicated a greater percentage than pre-survey who agreed with Q1 $(p=0.04)$. 
The homework problem-specific surveys revealed that the majority of students agreed strongly or somewhat that the two problems helped them understand how engineering statics/mechanics concepts apply globally to real-world scenarios and how engineers contribute to solving global challenges (Table 1). The majority of respondents also agreed the "Meet Aminah" problem increased their interest in global applications of engineering (Table 1).

Table 1. Percentage of respondents who agreed (strongly or somewhat)

\begin{tabular}{|l|c|c|}
\hline & $\begin{array}{c}\text { “Meet } \\
\text { Aminah” }\end{array}$ & $\begin{array}{c}\text { “Shoulder } \\
\text { Pole” }\end{array}$ \\
\hline $\begin{array}{l}\text { Q4: Helped understand how engineering statics/mechanics } \\
\text { concepts apply globally to real-world scenarios }\end{array}$ & $91 \%(48 / 53)$ & $85 \%(28 / 33)$ \\
\hline $\begin{array}{l}\text { Q5: Helped understand how engineers contribute to solving } \\
\text { global challenges }\end{array}$ & $85 \%(45 / 53)$ & $88 \%(29 / 33)$ \\
\hline $\begin{array}{l}\text { Q6: Increased my interest in global applications of } \\
\text { engineering }\end{array}$ & $62 \%(33 / 53)$ & $48 \%(16 / 33)$ \\
\hline
\end{tabular}

\section{Discussion}

In this preliminary study, we developed and implemented two globally-inspired homework problems in a traditional biomechanics course through a unique collaboration between Engineers Without Borders student members and faculty and an honors biomechanics student. We showed through indirect assessments (self-reported student survey responses) that these problems helped students understand how engineering statics/mechanics concepts apply globally to real-world scenarios and how engineers contribute to solving global challenges. We also showed that by the end of the course, $98 \%$ of survey respondents agreed that the relationship between engineering statics/mechanics concepts and global challenges was clear to them, an increase compared to the start of the semester. Because students were already interested in the applications of mechanics to global scenarios, as evidenced by their free responses and agreement with Q3, we did not see as high of gains in the problem-specific Q6. Additional comments noted in the free-responses indicated that students would like to go beyond calculating a value or justifying something that is already done and instead use their engineering toolset to solve a significant global problem.

\section{Path Forward}

Fall 2017 served as a pilot semester for implementation of two globally-inspired mechanics problems. An additional 15 honors students enrolled in Bioengineering Mechanics I in fall 2017 also collaborated with EWB students to create their own globally-focused application of statics and mechanics concepts. In future iterations of this study, we plan to implement more of these globally-inspired mechanics problems, incorporate direct assessments to evaluate whether these problems helped students attain our goals and the ABET outcomes, code the free responses to identify emergent themes, and compare those students enrolled in honors with those not enrolled in honors.

In conclusion, we have provided a framework to develop engineering problems with global context through a unique collaboration between biomedical engineering faculty and students and a campus-based global service-learning organization. Considering not all students have the opportunity to engage in experiential learning abroad, these problems allow instructors to expose more students to global applications of engineering design directly in the classroom. 


\section{Acknowledgements}

We thank Christopher Kitson, Natalie Muneses, and Tiange Zhang for their assistance in developing the problems.

\section{References}

[1] ABET, “Criteria for accrediting engineering programs, 2018-2019” [Online]. Available: www.abet.org [Accessed Jan. 26, 2018]

[2] B. K. Jesiek and K. Beddoes, "From Diplomacy and Development to Competitiveness and Globalization: Historical Perspectives on the Internationalization of Engineering Education,” in What is Global Engineering Education For?: The Making of International Educators, G. L. Downey and K. Beddoes, Ed. Morgan \& Claypool, 2010, pp. 45-76.

[3] National Academy of Engineering, Letter to Obama "Educating Engineers to Meet the Grand Challenges,” 2015 [Online]. Available: https://www.nae.edu/File.aspx?id=134217 [Accessed January 26, 2018]. 